\title{
Cost Analysis of Re-Sterilization Procedure of Re-Usable Devices in a Hospital
}

\author{
Naveen Kumar*1, Vrunda Lothlikar², Bryal D' Souza ${ }^{3}$, Usha Rani ${ }^{4}$, Swapna BV ${ }^{5}$ \\ ${ }^{1}$ Associate Professor, Department of Hospital Administration, Kasturba Medical College, MAHE, Manipal, Karnataka, INDIA. \\ ${ }^{2}$ Trainee, Prasanna School of Public Health, Manipal Academy of Higher Education, Manipal, Karnataka, INDIA. \\ ${ }^{3}$ Prasanna School of Public Health, Manipal Academy of Higher Education, Manipal, Karnataka, INDIA. \\ ${ }^{4}$ Prasanna School of Public Health, Manipal Academy of Higher Education, Manipal, Karnataka, INDIA. \\ 5 MMMC, Manipal Academy of Higher Education, Manipal, Karnataka, INDIA.
}

\begin{abstract}
Aim: To analyse the costs and recommend rectification in functioning of CSSD for qualitative delivery of patient care. Objectives: Assess the effectiveness of sterilization procedures and calculate the cost of re-sterilization of the batch which failed quality indicators tests. Method: It was observational prospective study done by random sampling technique for selecting sterilization cycles. In the present study, 1451 cycles of steam sterilization, 780 cycles of plasma sterilization and 312 cycles of ethylene oxide sterilization was observed. Results: The study revealed that 66 cycles of steam sterilization and 21 cycles of plasma sterilization failed the parameters of quality indicators. The total cost incurred on the hospital for re-sterilization was found to be Rs. 4,18,800/. Conclusion: The study emphasized the need of training of the CSSD technician for implementing the appropriate use of quality indicators for efficient sterilization. The study also focussed
\end{abstract}

on the need of in-house bank of frequently replenished spare parts and a planned maintenance program for sterilization equipment.

Key words: Sterilization Procedures, Training of Central sterile and supply department employees, Quality Indicators, Costing Analysis.

\section{Correspondence:}

Dr. P. Naveen Kumar, M.B.B.S. M.D. (Hospital Administration), Associate Professor, Department of Hospital Administration, Kasturba Medical College and Hospital, Manipal, - 576104 Karnataka, India.

Phone: +919019164899

Email: naveenpdr@yahoo.co.in

DOI: 10.5530/jyp.2018.10.24

\section{INTRODUCTION}

Efficient management of infrastructure and effective monitoring of disinfection and sterilization procedures is important for quality of patient care. Central sterile supply department (CSSD) is a service unit in a hospital that processes, issues, and controls the sterile stores supply to all departments of the hospital. ${ }^{1}$ The guidelines for disinfection and sterilization in Healthcare facilities, 2008, presents evidence based recommendations on preferred methods for cleaning, disinfection and sterilization of patient care medical services. ${ }^{2}$ Disinfection and sterilization are essential for ensuring that medical and surgical instruments do not transmit infectious pathogens to patients. Multiple studies in many countries have documented lack of compliance with established guidelines for disinfection and sterilization. Failure to comply has led to numerous outbreaks. ${ }^{2}$

Steam sterilization is most commonly used sterilization because it is safe, inexpensive and time saving. The outer layer of microorganisms is softened by the steam which coagulates the internal portion of microbes. Each item is subjected to temperature of $134^{\circ} \mathrm{C}$ for 3 minutes or $121^{\circ} \mathrm{C}$ for $15 \mathrm{~min}$ and pressure of 20 PSI for each cycle. ${ }^{3}$

Ethylene oxide is used in sterilization of items which are heat and moisture sensitive. ETO is a chemical agent that kills microorganisms by stopping the normal metabolisms of protein and reproductive process resulting in death of microbes. The gas concentration for each cycle is $100 \mathrm{mg} / \mathrm{dl}$. The aeration phase lasts for 12 hours. ${ }^{3}$ Cold Method: The temperature is $37^{\circ} \mathrm{C}$ and cycle duration is 5 hours. Warm Method: The temperature is $55^{\circ} \mathrm{C}$ and cycle duration is 2 hours. Hydrogen peroxide and formic acid generates Performic acid which is effective against wide range of bacteria and spores. It is used to sterilize heat or temperature sensitive articles such as rigid endoscopes. The cycle lasts for $45-70 \mathrm{~min}$ at $37-55^{\circ} \mathrm{C} .^{3}$

The sterilization procedure should be monitored routinely by using a combination of mechanical, chemical, and biological indicators to evaluate the sterilizing conditions and indirectly the microbiologic status of the processed items. ${ }^{4}$

The mechanical monitors for steam sterilization include the daily assessment of cycle time and temperature by examining the temperature record chart (or computer printout) and an assessment of pressure via the pressure gauge. The mechanical monitors for $\mathrm{EO}$ include time, temperature, and pressure recorders that provide data via computer printouts and gauges. ${ }^{4}$ Chemical indicators usually are either heat-or chemical-sensitive inks that change color when one or more sterilization parameters (e.g., steamtime, temperature, and/or saturated steam; EO - time, temperature, relative humidity and/or EO concentration) are present. ${ }^{4}$

Disinfecting before re-use and maintaining sterile surfaces on reusable devices involves high costs to hospitals. Using inexpensive technology will save money and time, but it requires extensive training. Good training will reduce the wastage cycles of sterilization in the machines. Hence, it was needed to identify the number of cycles that did not qualify for complete sterilization. The money spent for these cycles was computed. This much can be saved by providing effective training to the employees. The other causes leading to improper sterilization were also identified in the study. Hence the present study is planned to to assess the completion of sterilization procedures for infection free patient care and to calculate the cost of re-sterilization of the batch which failed quality indicators test.

This is an open access article distributed under the terms of the Creative Commons Attribution-NonCommercial-ShareAlike 4.0 License, which allows others to remix, tweak, and build upon the work non-commercially, as long as the author is credited and the new creations are licensed under the identical terms. 


\section{METHODOLOGY}

It is an observational and prospective study conducted in CSSD of a tertiary care medical college teaching hospital. Random sampling was used to select sterilization cycles - 1451 cycles of steam sterilization, 780 cycles of plasma sterilizer and 312 cycles of Ethylene oxide were observed. Checklist for mechanical, chemical and biological indicators were prepared as per guidelines mentioned in the guidebook to Accreditation standards for hospital (National Accreditation Board for Hospitals and Healthcare providers), $4^{\text {th }}$ Edition. Checklist for mechanical indicators consisted of assessment of duration of temperature, pressure and gas concentration maintained for each cycle. Checklist for chemical indicators was to observe the color change on paper strips of medical grade packing material. Checklist of biological indicators consisted of color change observed in cultures of Bacillus stearothermophillus and Bacillus Subtillis. If the biological indicators fail, then re-call process of dispatched sterilized items would be done from user departments and if chemical indicators failed then cost per unit of re-sterilization was calculated with help of variable cost and fixed cost. The prospective data of quality indicators were monitored from $24^{\text {th }}$ September 2016 to $31^{\text {st }}$ March 2017 and observations were noted in the checklist.

\section{RESULTS}

In the present study, 1451 cycles of steam sterilization, 780 cycles of plasma sterilization and 312 cycles of Ethylene oxide gas sterilization were observed in CSSD of tertiary care hospital to assess the effectiveness of sterilization procedures. Sterilization was performed by 5 autoclaves labelled as Steam sterilizer A, B, C, D, E, one Ethylene oxide gas sterilizer and one plasma sterilizer. The data was analysed with SPSS version 21.0 with descriptive statistics and frequency table. From the present study, we observed 280 cycles of steam sterilizer A, out of which, 261 cycles had compliance with parameters of quality indicators and 19 cycles had non-compliance. For Steam sterilizer B, out of 234 cycles, it was found that 198 cycles were in compliance with parameters of quality indicators and 36 cycles had failed to compliance.

For Steam sterilizer C, it was observed that out of 293 cycles, 282 cycles were in compliance with quality parameters and 11 cycles had non-compliance. For Steam sterilizer D, 313 cycles were observed, all cycles were found to be in compliance with parameters of quality indicators.

Similarly for Steam sterilizer E, 330 cycles were observed, all cycles had compliance with quality indicators. Out of 5 Steam sterilizers, equipment $B$ reported maximum number of non-compliance due to failed mechanical indicators i.e temperature and pressure parameters. Whereas equipments D and $\mathrm{E}$ have reported $100 \%$ compliance with the quality indicators, as shown in Table 1.1.

For plasma sterilization procedures, 780 cycles were observed, out which 759 cycles followed the compliance of quality indicators and 21 cycles failed to match the parameters of indicators, as shown in Chart 1.1. For Ethylene oxide gas sterilization, 312 cycles were observed, all the cycles were fulfilling the compliance of quality indicators.

The observations during our study showed for Steam Sterilizer A, out of 19 failed cycles, only 11 cycles had failed mechanical indicators and 8 cycles had failed chemical indicators, for Steam Sterilizer B, out of 36 failed cycles, 22 cycles had failed mechanical indicators and 14 cycles had failed chemical indicators, for Steam Sterilizer C, out of 11 failed cycles, 7 cycles had failed mechanical indicators and 4 cycles had failed chemical indicators, for Plasma Sterilizer, out of 21 failed cycles, 7 cycles had failed mechanical indicators and 14 cycles had failed chemical indicators as illustrated in bar graph Chart 1.2.

We went on to assess the loss to the hospital in terms of re-do of cycles in these failed cycles and the reasons for the failure of cycles.

Table 1.1: Steam sterilisation Cycles that were compliant and non-
compliant:
\begin{tabular}{ccccccc}
\multicolumn{1}{c}{ Steam A } & Steam B & Steam C & Steam D & Steam E \\
\hline Compliance & 261 & 198 & 282 & 313 & 330 \\
Non-compliance & 19 & 36 & 11 & 0 & 0 \\
\hline
\end{tabular}

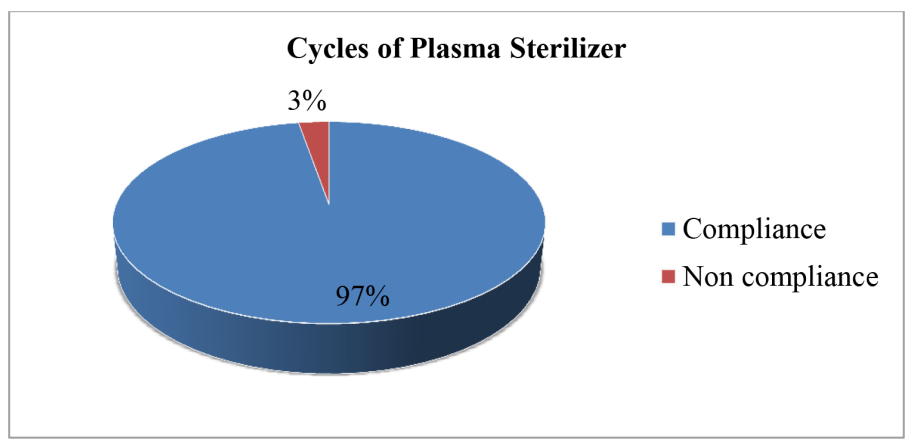

Chart 1.1: Showing compliant and non-compliant cycles in Plasma Sterilisation in this hospital:

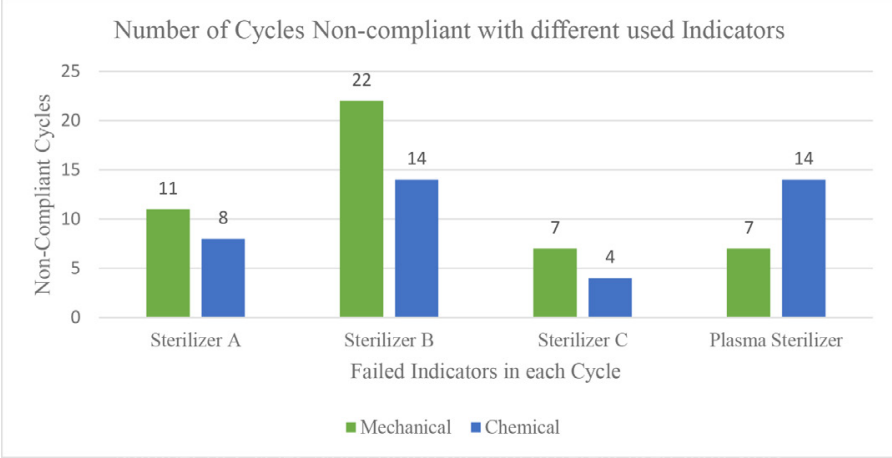

Chart 1.2: Showing the Sterilizers and their failed indicators in non-compliant cycles:

Cost of Sterilization incurred by hospital for one cycle of Steam Sterilization: Each_steriliser will accommodate in one cycle - 30 big sets, 60 medium sets and 100 small sets. These sets contain the instruments, devices that are re-used and the set is wrapped in loin cloth tagged with indicators. Cost that is incurred for re-sterilisation of each set is Rs. 40/-. When the mechanical indicators have failed then sterilization cycle was not initiated and packed unsterile items were loaded into another autoclave machine. When the Chemical indicators have failed then only chemical indicator paper strip over packed bundle was changed and all the bundles of unsterile items were re-loaded into same autoclave again.

Hence, the calculation considering only Chemical Indicator-failed cycles:

1. Number of big sets $\mathrm{x}$ unit re-sterilization cost $=30 \mathrm{X}$ Rs $40=$ Rs $1200 /$

2. Number of medium sets $\mathrm{x}$ unit re-sterilization cost $=60 \mathrm{X}$ Rs $40=$ Rs 2400/

3. Number of small sets $\mathrm{x}$ unit re-sterilization cost $=100 \mathrm{X}$ Rs $40=$ Rs 4000/ 
A total cost of Rs 7600/- is incurred to complete one cycle per steriliser. Cost of Re-sterilization incurred by hospital for one failed cycle of Steam Sterilizer A:

Number of failed cycles $\mathrm{x}$ Re-sterilization cost of each cycle $=8$ X 7600 $=$ Rs. $60,800 /-$

Cost of Re-sterilization incurred by hospital for one failed cycle of Steam Sterilizer B:

Number of failed cycles X Re-sterilization cost of each cycle $=14$ X 7600 = Rs. 106,400/-

Cost of Re-sterilization incurred by hospital for one failed cycle of Steam Sterilizer C:

Number of failed cycles X Re-sterilization cost of each cycle $=4$ X 7600 = Rs. 30,400/

Total cost of re-sterilization $=$ Rs 197,600/- (All above costs).

For Plasma sterilizer, cycles which failed chemical indicators had to be re-sterilized again. Each load of cycle of Plasma sterilizer consists of 10 Big sets +10 medium sets +50 small sets

Total cost of Sterilization for one cycle of Plasma sterilizer:

1. Number of Big sets X Re-sterilization cost of each set $=10$ X Rs. 350 $=$ Rs. 3500/

2. Number of Medium sets X Re-sterilization cost of each set $=10 \mathrm{X}$ Rs. $230=$ Rs. $2300 /$

3. Number of small sets $\mathrm{X}$ Re-sterilization cost of each set $=50 \mathrm{X}$ Rs. $200=$ Rs. $10,000 /$

Total cost of re-sterilization of one cycle plasma sterilizer $=3500+2300$ $+10,000=$ Rs $15800 /$

Total cost incurred by the hospital for re-sterilization in plasma sterilizer during study duration

Number of failed cycles X Cost of re-sterilization of each cycle $=14 \mathrm{X}$ $15800=$ Rs. 221,200/-.

Total cost incurred on hospital for re-sterilization procedures in CSSD during study duration:

Cost of re-sterilization of steam and plasma sterilization $=197,600+$ $221,200=$ Rs. $418,800 /-$

Further, root cause analysis to find the reasons for the equipment failing to comply with parameters of quality indicators showed, causative factors as delayed maintenance service from the manufacturer and non-availability of spare parts from local vendors on-time. The list of spare parts of autoclave equipment which are indispensable and which cannot be repaired and have to replaced and are non-available at local vendors are door gasket, pressure-valve, solenoid-valve, pneumatic-valve, heating coil, air filters and safety-valves.

\section{DISCUSSION}

In the present study, it was observed the guidelines mentioned in the Guidebook to Accreditation Standards for Hospital (NABH) $4^{\text {th }}$ edition were implemented in CSSD of tertiary care hospital to ensure infection free environment for patient care.

The objective elements from standard HIC 7 from NABH guidelines were incorporated in present study to monitor the effectiveness of sterilization procedures of CSSD and to enforce controls necessary for reducing the incidence of hospital acquired infections. Effectiveness of sterilization procedures were documented in daily registers, verified and signed by CSSD- In charge which is in compliance with guidelines of NABH $4^{\text {th }}$ Edition Standard HIC 7e. The validation tests for sterilization are carried out and documented regularly. ${ }^{5}$

We observed that none of the sterilizers had failed biological indicators tests, so the parameters for quality indicators were in compliance to standards of NABH guidelines. So no Re-call procedure was done during the study duration.

Mechanical and Chemical indicators have failed during observation of the study, but it did not disrupt the quality of patient care because the failed indicators were identified immediately at the end of the particular cycle and re-sterilization was done, these unsterile packed items were not dispatched to the user departments.

All 5 steam sterilizers had more number of failed mechanical indicators. The factors were evaluated through observations of functioning of CSSD, it was found that there was over utilization of steam sterilizers around 14 hours every day which lead to frequent wear and tear of spare parts like door gasket, solenoid-valve, gate-valve, pressure-valve and air-filters. Therefore, mechanical indicators for pressure and temperature could not be maintained as per standard guidelines. The present study also revealed that extra cost was incurred on the hospital for re-sterilization due to failed chemical indicators.

A study conducted in Iran during the period of 1997 to 2011, was aimed to determine the developing use of chemical and biological indicators for sterilization procedures of 21 district hospitals. This study concluded that there was significant improvement in sterilization control with the developing use of chemical indicators. ${ }^{6}$

As observed in the present study, Steam sterilizer B reported failed mechanical indicators for 22 cycles due to delayed maintenance service from the manufacturer. Further detailed observations revealed that Steam sterilizer A and C reported failed chemical indicators for 8 cycles and 4 cycles respectively due to worn out spare parts like pneumatic-valve, pressure-valve and solenoid-valve.

To ensure accurate maintenance of pressure, temperature and duration of cycle and also to reduce the financial burden of extra cost incurred on the hospital for re-sterilization procedures there is the need to provide the in-house bank of essential spare parts within CSSD of hospital so that these essential spare parts can be immediately replaced and uninterrupted functioning of sterilization procedures can be facilitated. Availability of these essential spare parts in- house of hospital for sterilizers under Comprehensive maintenance contract will reduce the lead time of procurement by the hospital as well as reduce the freight cost for the manufacturer.

The current preventive maintenance program for autoclave equipment is scheduled once in every 4 months. To reduce the probability of failures of compliance to quality indicators, it is essential to schedule the preventive maintenance after the sales service at predetermined intervals. To ensure uninterrupted functioning of autoclaves, it is necessary to schedule annual maintenance program as once in every 3 months so that the worn out spare parts can be detected and replaced at the earliest.

The present study also emphasized need for appropriate and timely training of CSSD staff for handling and operating the sterilizers as well as mandatory use of mechanical, chemical and biological indicators for effective sterilization of items. The training session should be planned to be conducted by the CSSD in-charge once in every month and by the service engineer of the manufacturer once in every 3 months, wherein both sessions should impart practical demonstration of operating techniques to all CSSD technicians.

A practical test should be conducted once in 3 months to evaluate the skills and knowledge of the CSSD technicians. The attendance of evaluation of skills and knowledge should be made mandatory as part of the performance appraisal for the CSSD technicians. The evaluation test will help hospital management to understand need for enforcement of training of CSSD technicians by the experts to facilitate proper functioning of CSSD of hospital. 


\section{CONCLUSION}

The findings of the current study, emphasized the need for regular and appropriate training of CSSD staff for using the equipment and mandating the use of mechanical, chemical and biological indicators appropriately to ensure infection free patient care.

The current study also emphasized on the need of in-house bank of essential and frequently replenished spare parts of sterilizers within the CSSD of the hospital to reduce the financial burden of extra cost of re-sterilization procedures incurred on the hospital and to ensure uninterrupted supply of sterile items for patients care.

The current study also focussed on the requirement of planned maintenance program of sterilization equipment for optimising the multifaceted operational and administrative aspects of the hospital.

\section{REFERENCES}

1. Banu A, Subhas GT. Central Sterile Supply Department- Need of the hour. $10^{\text {th }}$ ed. J Pub Health Med Res. Bangalore. J Pub Health Med Res. 2013;58-64.

2. Rutala W, Weber D. Guidelines for Disinfection and sterilization in healthcare facilities. 11th ed. CDC. North Carolina: Department of health and human services, USA; 2008;9-11

3. Joshi D, Joshi M. Hospital Administration. $1^{\text {st }}$ ed. Jaypee;. Pg 325-326

4. Sakharkar BM. Principles of Hospital Administration and Planning, $2^{\text {nd }}$ Edition, Jaypee Publishers, Pg 225-29.

5. National Accreditation Board for Hospitals and Healthcare providers (NABH). Guide Book to Accreditation standards for Hospitals (4 ${ }^{\text {th }}$ Edition). 2015;125-7.

6. Jabbari $H$, Alikah $H$, Alamdari S. Developing the use of quality indicators in sterilization practices. Iran J Public Health. 2012;41(7):64.

Article History: Submission Date : 26-09-2017 ; Revised Date : 07-10-2017; Acceptance Date : 12-11-2017.

Cite this article: Kumar N, Lothlikar V, Souza BD, Rani U, Swapna BV. Cost Analysis of Re-Sterilization Procedure of Re-Usable Devices in a Hospital. J Young Pharm. 2018;10(1):109-12. 